\title{
Optimum Directivity Patterns of Concentric Ring Arrays with Dipole Radiators for Wireless Communications
}

\author{
K. Kumar Naik \\ Associate Professor, Department of Electronics and Communication Engineering, \\ K L University, Greenfields, Veddeswaram, Guntur, Andhra Pradesh, India.
}

\begin{abstract}
In the literature survey concentric ring arrays using isotropic radiators are reported. Sufficient data is not available to optimization of the first sidelobe level, null to null beamwidth and directivity. In this paper, the author is proposed the problem of designing concentric ring arrays with practical radiating element. The practical radiating element is considered as dipoles to achieve optimum directional patterns for wireless communications. The numerically simulated patterns are computed and compared with the concentric isotropic ring arrays for different number of concentric rings.
\end{abstract}

\section{Keywords}

Concentric Ring Array, Concentric Dipole Ring Array, Isotropic Radiators, Wireless Communications

\section{INTRODUCTION}

Antennas are used in several domestic, military and civilian communications and radar applications. Several researchers have been proposed on linear arrays. In most of the conventional applications, linear arrays are used [1]. In the applications where angular symmetry is required ring arrays are used. A ring array consists of desired number of elements on a circular ring [2-3].

A ring arrays Designed by Redlich [4] for aircraft navigation. A technique for synthesizing a ring array that radiates a prescribed pattern in the plane of the ring is developed. Royer [5] has investigates the properties of ring arrays for which i) the serration in the horizontal field pattern are small, ii) the mean horizontal directive gain is greater than that of one of the monopoles from the array. Cheng et al. [6] derived the expressions for maximization of directive gain for circular and elliptical arrays.

Hussian et al. [7] reported a method for the synthesis of a very larger circular array in as deterministic manner. Space tapered highly thinned array are considered for the design and implementation. Steyskal [8] described a procedure to produce a desired power pattern.

Pencil beams are produced with main beam along the off axis investigated by Duncan [9]. The condition for maximizing the pattern gain function away from boresight is derived. Half power beam width and maximum gain are determined.

Preetham kumar et al. [10] has developed optimization technique for an antenna array, with a limited number of antenna array elements. The technique elaborates the design of a planar circular array with concentric rings of circular apertures with optimum elements radius for low sidelobe level.
Goto [11] reported a useful paper on the design for array antennas. Design is made in order to obtain high directivity and low sidelobes. The results are also presented. Harrrington [12] published excellent papers on the maximum realizable gain of arbitrary antenna enclosed by spherical surface.

Optimizations of small concentric ring arrays are designed by Huebner [13]. The desired pattern has either maximum directivity with minimum sidelobe level or maximum directivity with a specified sidelobe level. Hussain et al. [14] investigated clutter suppression for thinned array with phase only nulling. 16 concentric ring array directivity patterns are also presented.

A ring array produces first sidelobe level is about $-8 \mathrm{~dB}$. To overcome this concentric ring arrays are used and these are produces sidelobe level is about $-17.6 \mathrm{~dB}$ for isotropic radiating elements. But most of the researchers [15-22], they are reported to control the sidelobe levels using different techniques. These techniques are decreases the first sidelobe level only. But, faraway sidelobes are increases and directivity is decreases. Most of the communications and radar applications required more directivity.

In view of the above facts, intensive studies are made to analyse on the concentric dipole ring arrays. In the present work the analysis is carried out and the directional patterns are numerically simulated for concentric dipole ring arrays compared with concentric isotropic ring arrays.

\section{GENERAL ANALYSIS}

\subsection{Dipole Element Pattern}

The radiation pattern obtained is valid for any length of the dipole, provided it is centre-fed. Now centre-fed dipoles are characterized by the direction of the characteristics, which are similar to deformed figure of eight pattern.

Consider a dipole of length ' $2 L$ ' extending from $-L$ to $L$ along $z$-axis as shown in figure (1). Let its centre be at $z=0$. When the length of the dipole is very small compared to $\lambda$, the current is approximated to be a constant. If its length is less than $\lambda / 4$, the current distribution can be approximated to be triangular. For other lengths, it should be exact and it can be determined considering the antenna as an open ended transmission line.

However, the current distribution is found to be sinusoidal for lengths of order $\lambda / 2$. For equilengths, it will be a low distorted sinusoidal distribution. Here, the sinusoidal distribution is assumed to find out the element patterns.

The current distribution is given by 
$\mathrm{I}(\mathrm{z})=\mathrm{I}_{\mathrm{m}} \operatorname{sink}(\mathrm{L}-\mathrm{z})$,

$\mathrm{I}(\mathrm{z})=\mathrm{I}_{\mathrm{m}} \operatorname{sink}(\mathrm{L}-\mathrm{z}), \quad$ for $\quad \mathrm{z}>0$

$\mathrm{I}(\mathrm{z})=\mathrm{I}_{\mathrm{m}} \operatorname{sink}(\mathrm{L}+\mathrm{z})$ for $\mathrm{z}<0$ and

$\mathrm{I}(\mathrm{z})=0$, for $\mathrm{z} \geq \pm \mathrm{L}$

Here, $\quad k=\frac{2 \pi}{\lambda}$, is wave number and $I_{m}=$ maximum current

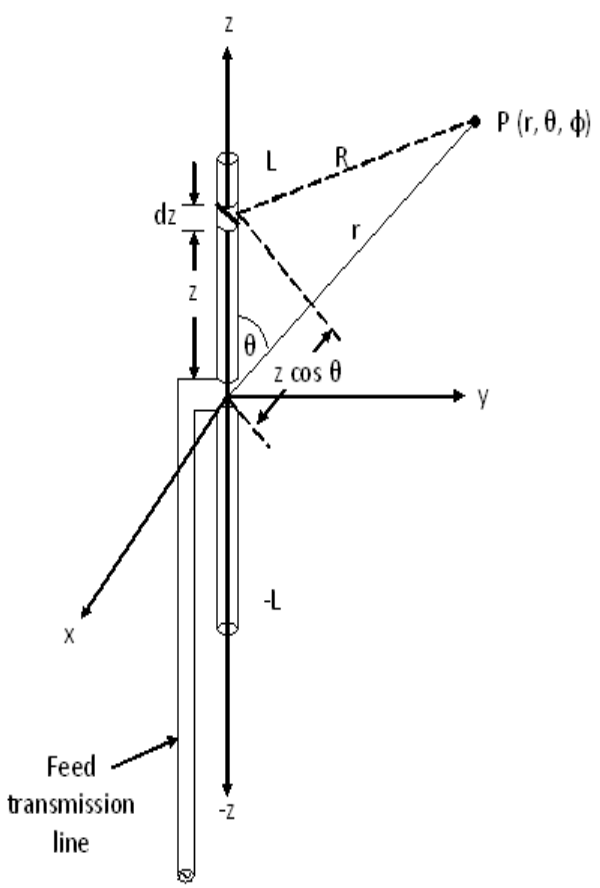

Fig.1 Typical Geometry of Dipole Element

The vector magnetic potential due to the elementary length $d z$ at a point $P$ in free space is given by

$$
\mathrm{dA}_{\mathrm{z}}=\frac{\mu \mathrm{I}(\mathrm{z}) \exp (-\mathrm{jkR})}{4 \pi \mathrm{R}} \mathrm{dz}
$$

The total vector magnetic potential is then given by

$$
\begin{aligned}
A_{z}=\frac{\mu}{4 \pi} \int_{-L}^{L} \frac{I(z) \exp (-j k R)}{R} d z \\
=\frac{\mu}{4 \pi}\left[\begin{array}{l}
\int_{-L}^{0} \frac{I_{m} \sin \beta(L+z) \exp (-j k R)}{R} d z+ \\
\int_{0}^{L} \frac{I_{m} \sin \beta(L-z) \exp (-j k R)}{R} d z
\end{array}\right]
\end{aligned}
$$

For the distant or radiation field, $R$ is considered to be very large and $R \approx r$. However, in the phase vector, the difference between $R$ and $r$ is important and it can be written as
$\mathrm{R}=\mathrm{r}-\mathrm{z} \cos \theta$

$A_{z}=\frac{\mu I_{m} \exp (-j k r)}{2 \pi r}\left[\int_{0}^{L} \sin k(L-z) \cos (k z \cos \theta)\right]$

Evaluating the integral and simplifying, we get,

$$
A_{z}=\frac{\mu I_{m} \exp (-j k r)}{2 \pi k r}\left[\frac{\cos (k L \cos \theta)-\cos k L}{\sin ^{2} \theta}\right]
$$

The spherical components of the magnetic potential are

$$
\left.\begin{array}{l}
\mathrm{A}_{\mathrm{r}}=\mathrm{A}_{\mathrm{z}} \cos \theta, \\
\mathrm{A}_{\theta}=-\mathrm{A}_{\mathrm{z}} \sin \theta \quad \text { and } \\
\mathrm{A}_{\phi}=0
\end{array}\right\}
$$

The magnetic field strength $\mathbf{H}$ is obtained through the relation

$\mu \mathrm{H}=\nabla \times \mathbf{A}$

The current is entirely in $z$ - direction and for radiation field $r$ is assumed to be very large. Therefore,

$$
\begin{aligned}
& \mu \mathrm{H}_{\phi}=-\frac{\mathrm{dA}_{\mathrm{z}}}{\mathrm{dr}} \sin \theta \\
& \mathrm{H}_{\phi}=\frac{\mathrm{jI} \mathrm{I}_{\mathrm{m}} \exp (-\mathrm{jkr})}{2 \pi \mathrm{r}}\left[\frac{\cos (\mathrm{kL} \cos \theta)-\cos \mathrm{kL}}{\sin \theta}\right]
\end{aligned}
$$

The electric field strength for the radiation field is

$$
\mathrm{E}_{\theta}=\eta \mathrm{H}_{\phi}
$$

Here, $\eta=120 \pi$

$E_{\theta}=\frac{j I_{m} 60 \exp (-j k r)}{r}\left[\frac{\cos (k L \cos \theta)-\cos k L}{\sin \theta}\right]$

\subsection{Pattern of Concentric Dipole Ring Arrays}

The elements are arranged in planar circular arrays containing concentric circular dipole rings is shown in figure (2), which differ in radius and number of elements. A concentric dipole ring array with ring $m$ having $N_{m}$ elements and a radius of $r_{m}$. The distance between elements on ring $m$ is given by $d_{m}$ and distance between any two elements is constant. 


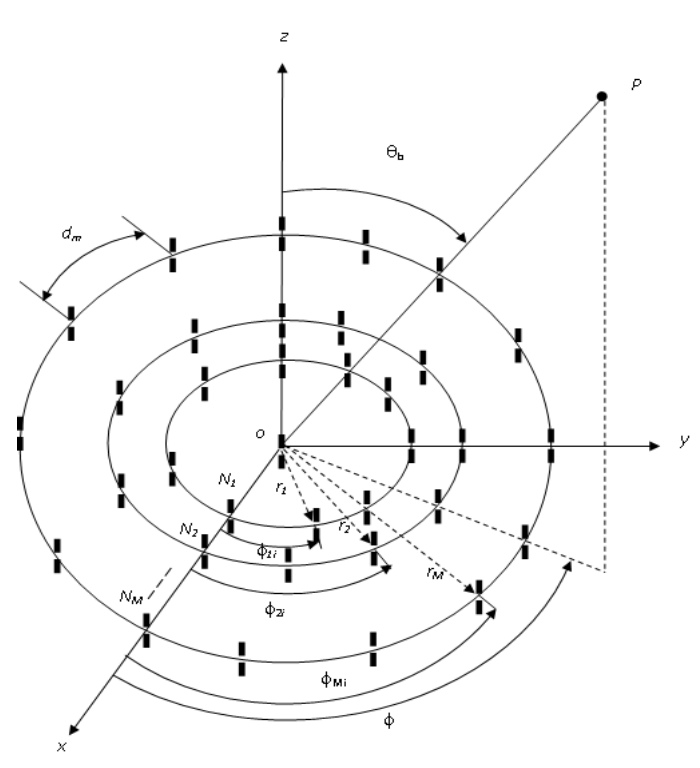

Fig. 2 Typical Geometry of Concentric dipole ring array

The far-field pattern of the concentric dipole ring array with a single element at the centre is given by

$$
E_{d}=E_{\theta} \times\left(\begin{array}{l}
I_{0}+\sum_{m=1}^{N_{M}} \sum_{i=1}^{N_{m}} I_{m i} \times \\
\exp \left[j k r_{m} \sin \theta \cos \left(\phi-\phi_{m i}\right)+j \alpha_{m i}\right]
\end{array}\right)
$$

Here,

$E_{\theta}$ is normalized element pattern of dipole element $N_{M}$ is number of rings,

$N_{m}$ is number of elements on the $m^{\text {th }}$ ring,

$I_{m i}$ is current excitation of the $i^{\text {th }}$ element of the $m^{\text {th }}$ ring, $I_{0}=1$ is considered,

$\mathrm{k}=\frac{2 \pi}{\lambda}, \lambda$ is wavelength,

$r_{m}=$ radius of the ring $m$,

$\left(\mathrm{x}_{\mathrm{i}}, \mathrm{y}_{\mathrm{i}}\right)=\left(\mathrm{r}_{\mathrm{i}} \cos \phi_{\mathrm{mi}}, \mathrm{r}_{\mathrm{i}} \sin \phi_{\mathrm{mi}}\right)=$ location of the element $i$,

$\theta=$ Elevation angle, $\phi=$ Azimuth angle,

$\phi_{\mathrm{mi}}=\frac{2 \pi(\mathrm{i}-1)}{\mathrm{N}_{\mathrm{m}}}, \quad$ where, $\mathrm{m}=1,2, \ldots, \mathrm{M} ; \mathrm{i}=1,2, \ldots, \mathrm{N}_{\mathrm{m}}$

$\phi_{\mathrm{mi}}=$ Angular location of element $i$ of the $m^{\text {th }}$ ring,

$d_{m}=$ Interelement spacing of $m^{\text {th }}$ ring

$\mathrm{N}_{\mathrm{m}}=\frac{2 \pi \mathrm{r}_{\mathrm{m}}}{\mathrm{d}_{\mathrm{m}}}, \mathrm{r}_{\mathrm{m}}=\frac{\mathrm{m} \lambda}{2}, \mathrm{~d}_{\mathrm{m}}=\frac{\lambda}{2}$

If an additional phase is applied for each element, it is possible to scan the beam. Such a phase function is represented by

$\alpha_{\mathrm{mi}}=\exp \left[\mathrm{jk} \mathrm{r}_{\mathrm{m}} \sin \theta_{\mathrm{s}} \cos \left(\phi_{\mathrm{s}}-\phi_{\mathrm{m}}\right)\right]$

Here, $\left(\theta_{\mathrm{s}}, \phi_{\mathrm{s}}\right)=$ Steering angle,

In the above expression (14), consider the elements of same weight in amplitude, and phase. The field pattern is independent of $\phi$, and it is dominated by the principle terms for each ring, where the residuals are negligible. The field pattern for a ring array is expressed in terms of Bessel function. The summation term in the above expression is the array factor. For the array of present interest, we have the farfield radiation patterns for concentric dipole ring array is expressed as

$$
\mathrm{E}_{\mathrm{d}}=\mathrm{E}_{\theta} \times\left(1+\sum_{\mathrm{m}=1}^{\mathrm{M}} \mathrm{I}_{\mathrm{mi}} \mathrm{N}_{\mathrm{m}} \mathrm{J}_{0}\left(\mathrm{k} \mathrm{r}_{\mathrm{m}} \sin \theta\right)\right)
$$

Here, $\mathrm{E}_{\theta}$ is normalized dipole element pattern is

$$
\mathrm{E}_{\theta}=\left[\frac{\cos (\mathrm{kL} \cos \theta)-\cos \mathrm{kL}}{\sin \theta}\right]
$$

\subsection{Directivity}

The directivity is evaluated for concentric dipole ring arrays' using the following expression is given by [1]

$$
\mathrm{D}=\frac{4 \pi\left|\mathrm{E}_{\mathrm{d}}\left(\theta_{\mathrm{s}}, \phi_{\mathrm{s}}\right)\right|^{2}}{\int_{0}^{2 \pi} \int_{0}^{\pi}\left|\mathrm{E}_{\mathrm{d}}(\theta, \phi)\right|^{2} \sin \theta \mathrm{d} \theta \mathrm{d} \phi}
$$

The directivity is computed for concentric ring arrays using dipole radiators from the expression (17). To obtain the maximum directivity, considered uniform amplitude, uniform phase, and spacing between the elements to be $\lambda / 2$.

\section{RESULTS AND DISCUSSIONS}

Using the expressions (17), the radiation patterns are numerically evaluated for the concentric dipole ring arrays. In expression (17), the element pattern $E_{\theta}$ to be considered as isotropic element $\left(\mathrm{E}_{\theta}=1\right)$, then that produce the radiation pattern of concentric isotropic ring arrays. In figures (3-10), the directional patterns of the concentric dipole ring arrays and concentric isotropic ring arrays with different rings are presented.

From the figure (3) shown the comparison of two concentric dipole and isotropic rings. Considered as a two concentric rings $(M=2)$, found that sidelobe level is $-20.76 \mathrm{~dB}$ for dipole radiators and the sidelobe level is $-17.6 \mathrm{~dB}$ for isotropic radiator, null to null beamwidth is 60.68 degrees for both cases. Similarly, the patterns are evaluated for different concentric rings $(M=3,5,7,9,11,17$, and 21) and the data is presented in table 1 for concentric dipole rings.

Using the expression (19) the directivity is evaluated for the concentric dipole ring arrays and the data is presented in the table 1 .

In the figure (11) shows the directivity increases while increasing the number of concentric dipole rings. Similarly, a figure (12) show the null to null beamwidth is reduces while increasing the number of concentric dipole rings. 
International Journal of Computer Applications (0975 - 8887)

Volume 69-No.12, May 2013

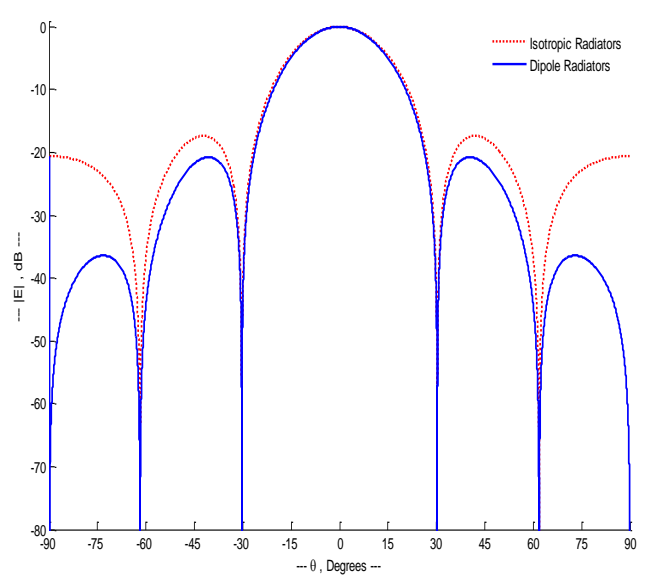

Fig. 3 Patterns for a Isotropic and Dipole Concentric Ring Arrays of Number of Rings, $M=2$

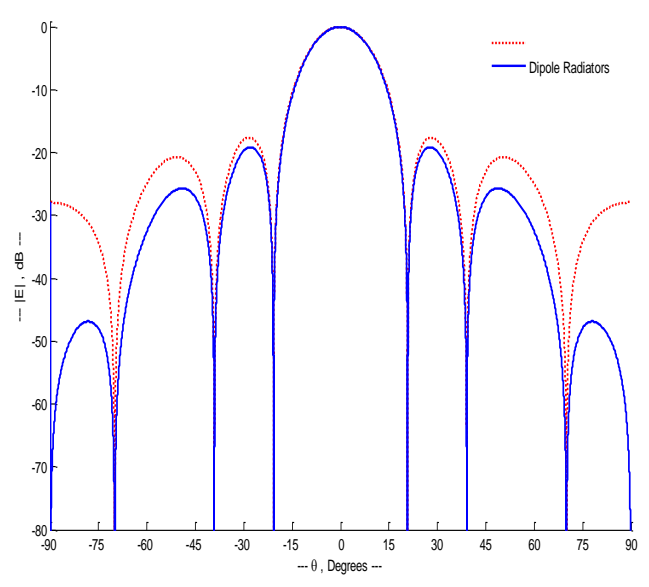

Fig. 4 Patterns for a Isotropic and Dipole Concentric Ring Arrays of Number of Rings, $M=3$

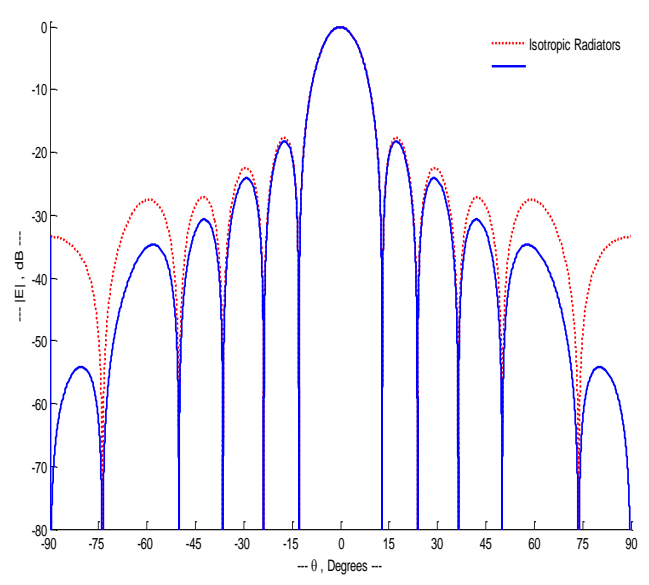

Fig. 5 Patterns for a Isotropic and Dipole Concentric Ring Arrays of Number of Rings, $M=5$

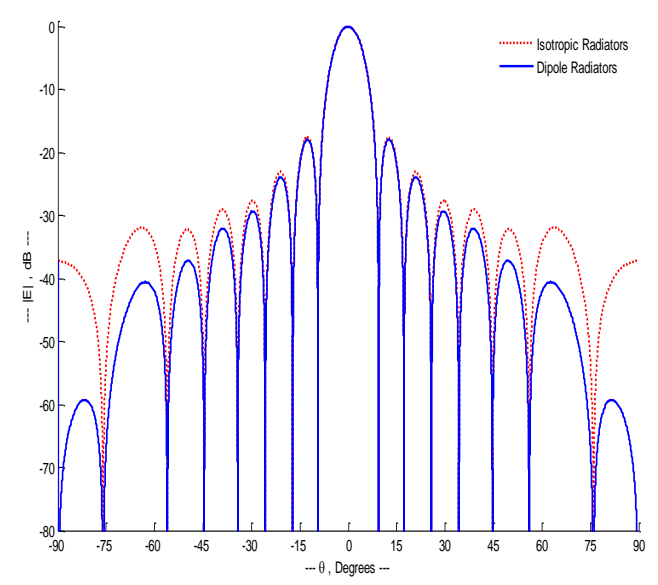

Fig.6 Patterns for a Isotropic and Dipole Concentric Ring Arrays of Number of Rings, $M=7$

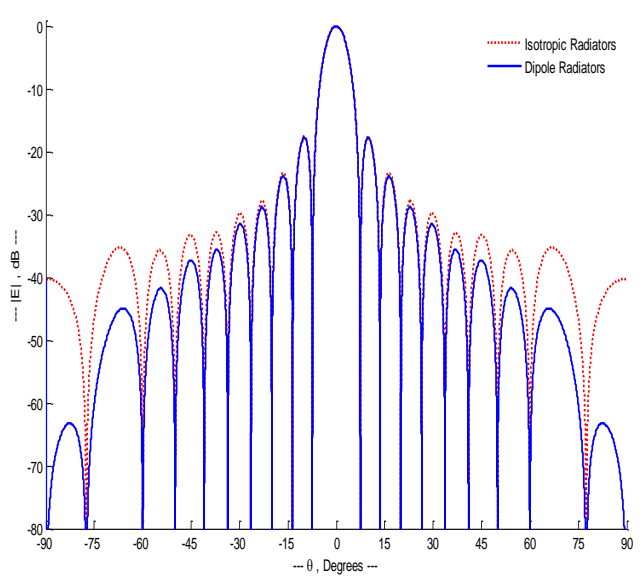

Fig. 7 Patterns for a Isotropic and Dipole Concentric Ring Arrays of Number of Rings, M=9

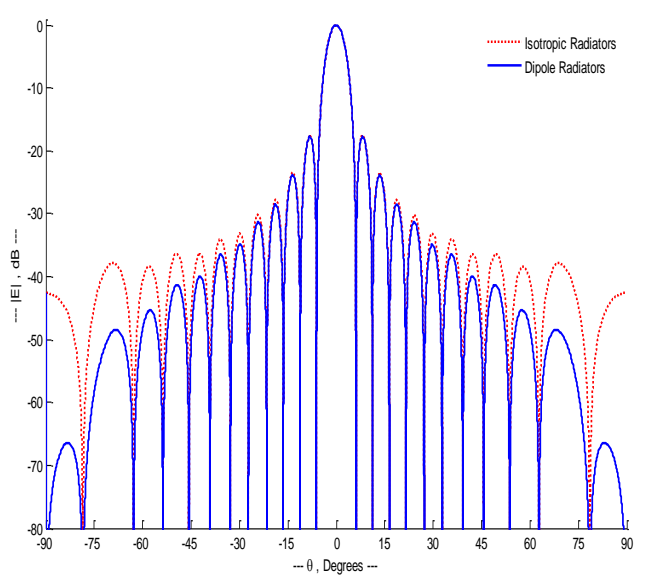

Fig. 8 Patterns for a Isotropic and Dipole Concentric Ring Arrays of Number of Rings, $M=11$

20 


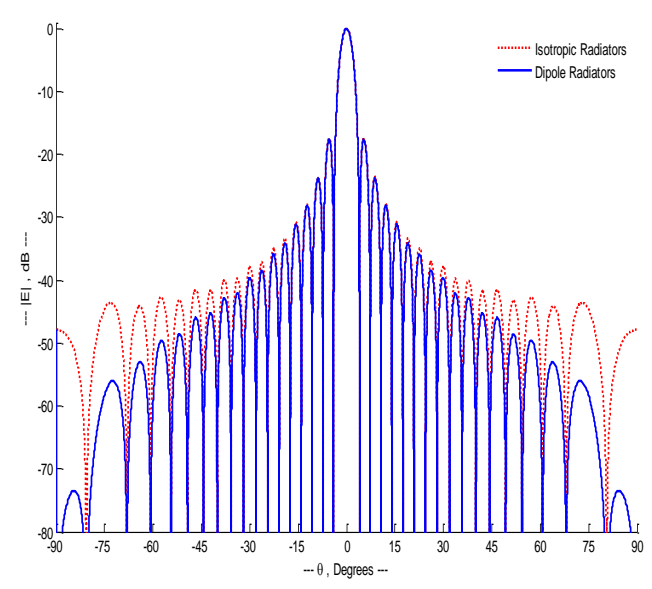

Fig. 9 Patterns for a Isotropic and Dipole Concentric Ring Arrays of Number of Rings, $M=17$

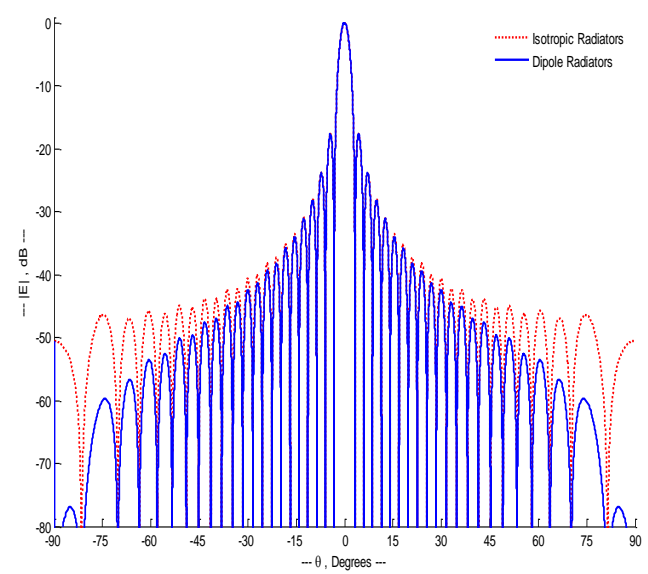

Fig.10 Patterns for a Isotropic and Dipole Concentric Ring Arrays of Number of Rings, $M=21$

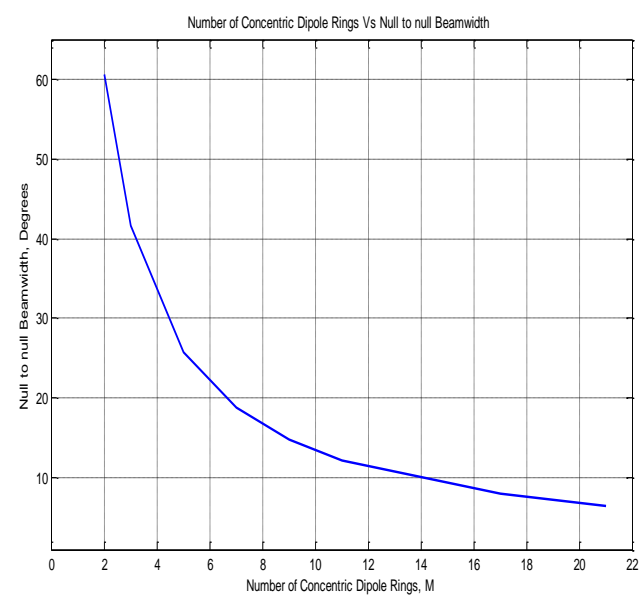

Fig. 11 Null to null beamwidth variations versus different number of concentric dipole rings

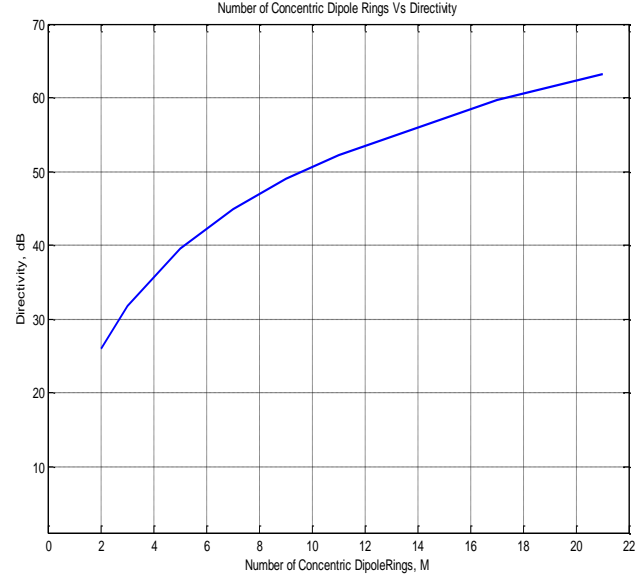

Fig. 12 Directivity variations versus different number of concentric dipole rings

Table 1. Pattern characteristics of concentric dipole ring arrays

\begin{tabular}{|c|c|c|c|c|}
\hline $\begin{array}{c}\text { Number } \\
\text { of rings } \\
(\mathbf{M})\end{array}$ & $\begin{array}{c}\text { First } \\
\text { sidelobe } \\
\text { level in } \\
\text { dB }\end{array}$ & $\begin{array}{c}\text { Last } \\
\text { sidelobe } \\
\text { level in } \\
\mathbf{d B}\end{array}$ & $\begin{array}{c}\text { Null-to-null } \\
\text { beamwidth } \\
\text { in degrees }\end{array}$ & $\begin{array}{c}\text { Directivi } \\
\text { ty } \\
\text { in dB }\end{array}$ \\
\hline 2 & -20.76 & -36.42 & 60.68 & 25.95 \\
\hline 3 & -19.14 & -46.89 & 41.66 & 31.70 \\
\hline 5 & -18.19 & -54.19 & 25.80 & 39.58 \\
\hline 7 & -17.90 & -59.30 & 18.78 & 44.96 \\
\hline 9 & -17.78 & -63.23 & 14.78 & 49.06 \\
\hline 11 & -17.71 & -66.43 & 12.20 & 52.26 \\
\hline 17 & -17.63 & -73.52 & 7.98 & 59.66 \\
\hline 21 & -17.62 & -77.05 & 6.50 & 63.24 \\
\hline
\end{tabular}

\section{CONCLUSIONS}

It is evident from the results that the sidelobe level of the concentric ring array dipole radiators is decreases compared to the concentric ring array of isotropic radiators without deteriorating the null to null beamwidth. The faraway sidelobe levels of the concentric dipole ring arrays are very less compared to the concentric isotropic ring arrays. While increasing the number of concentric rings the beamwidth is reduced and directivity is increased. These results are mainly used for wireless communications in broadband and narrowband applications. The data is helpful for array antenna designers to implement the real time applications.

\section{REFERENCES}

[1] G.S.N. Raju, “Antennas and Wave Propagation”, Pearson Education (Singapore) Pte., Ltd., New Delhi, 2005. 
[2] M.T. Ma, "Theory and Applications of Antenna Arrays", Johnwiley and Sons, New York, 1974.

[3] Robert S. Elliott, "Antenna theory and Design”, PrenticeHall, USA,1981.

[4] R.W. Redlich, "Sampling synthesis of ring arrays", IEEE Transaction on Antennas and Propagation, pp. 116-118, January 1970.

[5] G.M.Royer, "Directive gain and impedance of ring array of antenna", IEEE Transaction on Antennas and Propagation, vol. AP -14, no.5, pp. 566-573, September 1966.

[6] D.K. Chang and F.I. Tseng, "maximization of directive gain for circular and elliptical arrays", proc. IEE, Vol. 114, no. 5, pp.589-594, May1967.

[7] M.A.Hussain, W.Lin and M.McKee, "Representation of continuous circular aperture as a set of discrete ring for a prescribed radiation patterns", IEEE Transactions on Antennas and Propagation, Vol. 27, No. 5, PP 38723875, September 1987.

[8] H. Steyskal, "On Antenna Power Pattern synthesis", IEEE Transactions on transaction antennas propagation, Vol.AP-18, pp. 123-124, Jan 1970.

[9] J.W. Duncan, "Maximum off-Axis Gain of Pencil Beams," Proceedings of the IEEE, pp. 1791-1792, October 1969.

[10] B. Preetham kumar and G. R Branner, "Design of low sidelobe circular ring arrays by element radius optimization," Proceeding of the IEEE Transactions on Antennas and Propagation society symposium, pp. 20322035, 1999.

[11] Naohisa Goto, "A synthesis of array antennas high directivity and low sidelobes", IEEE Transactions on Antennas and Propagation, Vol. AP-20, No. 4, pp.427431, July 1972.

[12] R.F.Harringtion, "On the gain and beamwidth of directional antennas", IRE Transactions on Antennas and Propagation, Vol. AP-6, pp. 219-224, July 1958.

[13] M.D.A. Huebner, "Design and optimization of small concentric ring arrays," Proceeding of the IEEE Transactions on Antennas and Propagation, pp. 455-458, May 1978.
[14] Hussain et al., "Clutter suppression for thinned array with phase only nulling", United States Patent, No. 5515060, May 1996.

[15] C.O. Streans and A.C. Stewart, "An investigation of concentric ring arrays with low sidelobes", IEEE Transactions on Antennas and Propagation, Vol. AP-13, pp. 856-863, November, 1965.

[16] Lawrence S. Biller and Gerald E. Friedman, "Optimization of radiation patterns for an array of concentric ring sources", IEEE Transactions on Audio and Electrostatics, Vol. AU-21, No. 1, pp.57-61, February 1973.

[17]M. Dessouky, H. Sharshar, Y. Albagory, "Effecient sidelobe reduction technique for small-sized concentric circular arrays," Progress in Elecrtomagnetics Research, PIER 65, pp. 187-200, 2006.

[18] Yasser Attia Albagory, Moawad Dessouky, Hamdy Sharshar, "An approach for low sidelobe beamforming in uniform concentric circular arrays," Wireless Personal Communication, Springer, Vol. 43, pp. 1363-1368, December 2007.

[19] Randy L. Hupt "Optimized element spacing for low sidelobe concentric ring arrays" IEEE Transactions on Antennas and Propagation, Vol.AP-56, pp.266-268, January, 2008.

[20] Randy L. Hupt “Thinned concentric ring arrays," IEEE Transactions on Antennas and Propagation, pp. 1-4, 2008.

[21] N. Pathak, P. Nanda, and G.K. Mahanthi, "Synthesis of thinned multiple concentric circular ring array antennas using particle swarm optimization" Journal of Milli Terahz Waves, Springer, pp. 709-716, April 2009.

[22] D. Mandal, S.P Ghoshal, A.K. Bhattacharjee, "Design of concentric circular antenna array with central element feeding using particle swarm optimization with constriction factor and inertia weight approach and evolutionary programming technique," Journal Infrared Milli Terahz Waves, Springer, pp. 667-680, March 2010. 